\title{
Dresdner Dermatologische Demonstration 2015 - zugleich Tagung der Sächsischen Dermatologischen Gesellschaft am 21. März 2015*
}

\author{
Dresden Dermatology Demonstration 2015 - Meeting of the Saxonian Society of Dermatology
}

March 21, 2015

Autoren

Institut

\section{G. Hansel, A. Koch, U. Wollina}

Klinik für Dermatologie und Allergologie am Krankenhaus Dresden-Friedrichstadt, Städtisches Klinikum,

Akademisches Lehrkrankenhaus der TU Dresden

\section{Bibliografie}

DOI http://dx.doi.org/

10.1055/s-0034-1391931

Akt Dermatol 2015; 41: 176-184

(c) Georg Thieme Verlag KG

Stuttgart · New York

ISSN 0340-2541

\section{Korrespondenzaddresse}

\section{Prof. Dr. med. Uwe Wollina}

Klinik für Dermatologie und

Allergologie am Krankenhaus

Dresden-Friedrichstadt,

Städtisches Klinikum,

Akademisches Lehrkrankenhaus

der TU Dresden

Friedrichstraße 41

01067 Dresden

wollina-uw@khdf.de

\section{Generalisierte eruptive Histiozytose (Histiozytome)}

\section{A. Bennewitz, S. Großmann, J. Schönlebe}

Anamnese: Der 60-jährige Patient wurde in unsere Klinik aufgrund von progredient auftretenden, mäßig juckenden Papeln eingewiesen. Er gab eine Bestandsdauer der Effloreszenzen von ca. $1 / 4$ Jahr an. Initial waren die oberen Extremitäten betroffen. Im Verlauf war eine Generalisierung mit Beteiligung des gesamten Integumentes zu beobachten. Ambulant zweimal durchgeführte orale Prednisolonstöße wären ohne therapeutisches Ansprechen geblieben.

Hautbefund: Es fanden sich am gesamten Integument disseminierte, follikulär gebundene, dezent erhabene, hautfarbene bis rötliche Papeln ( $\bullet$ Abb.1). Die behaarte Kopfhaut, Palmae, Plantae und Schleimhäute waren frei. Im Gesicht war eine flächige erythematöse Infiltration zu sehen.

Histologie: Im Papillarkörper und oberen Korium an mehreren Stellen ein teils rasenartiges, teils knotiges Infiltrat aus überwiegend spindeligen und epitheloiden CD68-positiven histiozytären Zellen mit Beimengungen mehrerer mehrkerniger Riesenzellen vom Fremdkörper- und Langerhanstyp ( $\bullet$ Abb.2a und b). Eingestreut sieht

\footnotetext{
* Vorsitz: Prof. Dr. U. Wollina

Berichterstatter: Frau Dr. G. Hansel, Dr. A. Koch,

Prof. Dr. U. Wollina

Histopathologie: Frau Dr. J. Schönlebe,

Prof. Dr. G. Haroske

Klinische Fotodokumentation: Frau R. Herz

Plenarvorträge:

PD Dr. M. Görnig, Aue: Polypharmazie -

gibt es einen Ausweg?

Dr. M. Klare, Zechlin: Sekundäres Beinlymphödem Prof. Dr. L. Kowalzick, Plauen/Vgtl.: Moderne onkologische Therapie: Fortschritte und Limits

Dipl.-Biol. A. Weck-Heimann, Dresden: Die Sandmücken kommen!

Prof. Dr. M. Maurer, Berlin: Urtikaria-Update
}

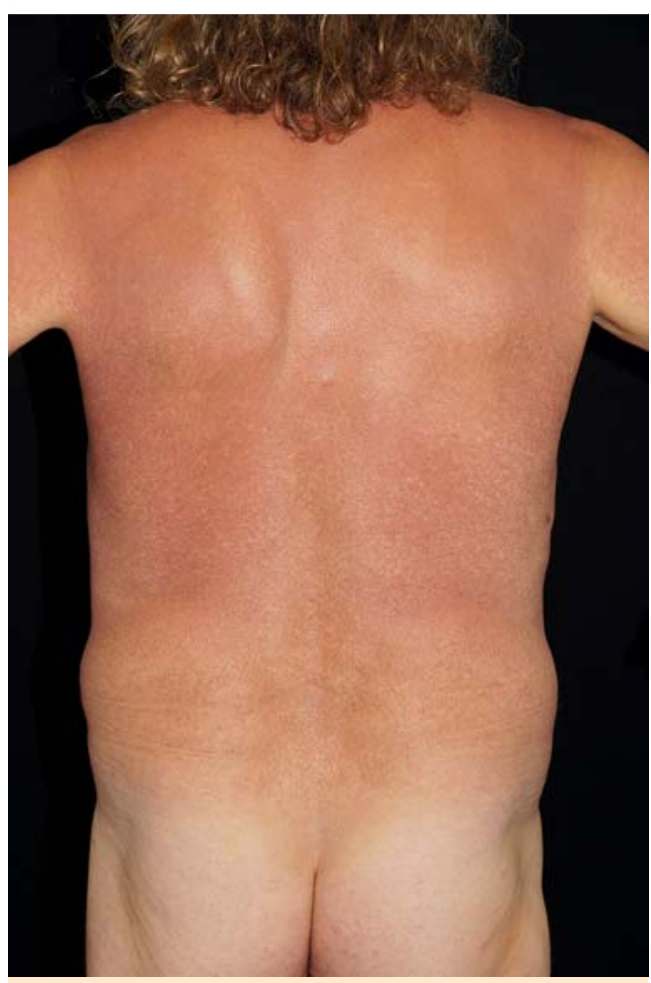

Abb. 1 Multiple flache Papeln am Rumpf bei generalisierter eruptiver Histiozytose.

man in wechselnder Zahl Lymphozyten, neutrophile Granulozyten und einzelne Eosinophile sowie Mastzellen. Die beschriebenen histiozytären Zellen exprimieren weder CD1a noch S100. Man sieht jedoch eine Vermehrung/Aktivierung ortsständiger (vor allem intraepidermaler) Langerhanszellen. Abschnittsweise hat das beschriebene Infiltrat angedeutet granulomatösen Charakter. Laborbefunde: CRP-Wert 52,6 mg/l (normal $<5 \mathrm{mg} / \mathrm{l}$ ).

Therapie und Verlauf: Unter dem kombinierten Therapieregime von Creme-PUVA und topischen Steroiden der Klasse II-III konnte in wenigen 


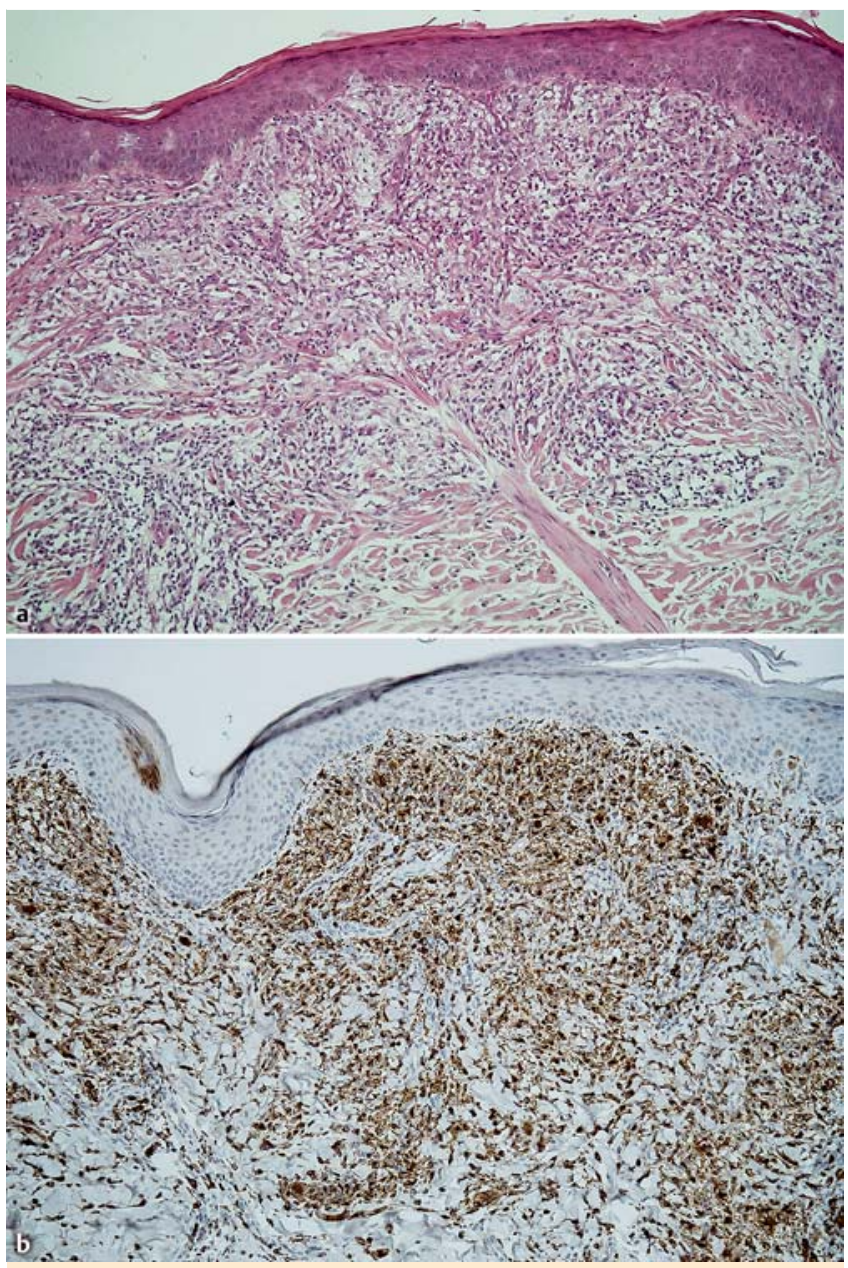

Abb.2 Histologie der generalisierten eruptiven Histiozytose. a Hämatoxylin-Eosin (×10), b CD68-Immunperoxidasefärbung (×10).

Tagen ein deutlicher Rückgang der Infiltration mit einem Abflachen der Papeln dokumentiert werden. Ambulant wurde folgend in wöchentlichem Turnus eine Sole-UVA-Therapie fortgeführt. Nach einer 4-monatigen Behandlungsdauer war eine komplette Abheilung erreicht.

Kommentar: Die Histiozytosen werden in 4 Typen klassifiziert: Typ I - Langerhanszell-Histiozytose, Typ Ila - Histiozytosen mit Zellen aus der dermalen dendritischen Linie, Typ IIb - Histiozytosen, die weder Langerhanszellen noch dermale Dendrozyten beinhalten, und Typ III - maligne histiozytische Erkrankungen [1]. Die generaliserte eruptive Histiozytose (generalisiertes eruptives Histiozytom) ist ein äußerst seltenes Krankheitsbild, welches zu den Nicht-Langerhanszell-Histiozytosen (Typ IIb) gemeinsam mit den Erkrankungen Xanthoma disseminatum, progressive noduläre Histiozytose und multizentrische Retikulohistiozytose zählt [2]. Die erstmals von Winkelmann und Muller 1963 beschriebene Erkrankung gehört zum Formenkreis der Makrophagenerkrankungen [3].

Es entstehen generalisiert, oft eruptiv, disseminierte, hautfarbene bis bläulich-rötliche Papeln. In Ausnahmefällen können die Schleimhäute mitbetroffen sein. Teils können nach Abheilung hyperpigmentierte Makulae sichtbar bleiben.

Histologisch setzen sich die Papeln aus vakuolisierten Histiozyten zusammen. Die Zellen exprimieren CD68, sind aber negativ für CD34, S100, CD1a und Faktor XIIIa. Meist persistieren die Hautveränderungen für Monate bis Jahre, bevor eine spontane
Abheilung zu beobachten ist. Therapeutisch kommt eine PUVATherapie in Betracht [4]. Orales Isotretinoin wurde in einem Fall erfolgreich eingesetzt [5].

Da die Erkrankung ein paraneoplastisches Syndrom darstellen kann, ist eine langfristige Verlaufsbeobachtung mit Kontrollen des Blutbildes empfehlenswert.

\section{Literatur}

1 Chu AC. Histiocytosis. In: Burns T, Breathnach S, Cox N, Griffiths C, eds, Rook's Textbook of Dermatology. 8th ed. Oxford: Blackwell Publishing; 2010: 55.1 - 55.33

2 Seward JL, Malone JC, Callen JP. Generalized eruptive histiocytosis. J Am Acad Dermatol 2004; 50: 116-120

3 Winkelmann RK, Muller SA. Generalized eruptive histiocytoma: a benign papular histiocytic reticulosis. Arch Dermatol 1963; 88: 586 - 596

4 Lan Ma H, Metze D, Luger TA, Steinhoff M. Successful treatment of generalized eruptive histiocytoma with PUVA. J Dtsch Dermatol Ges 2007; 5: $131-134$

5 Kwinter J, DeKoven J. Generalized eruptive histiocytoma treated with isotretinoin. J Cutan Med Surg 2009; 13: 146-150

\section{Großzelliges anaplastisches CD30-positives T-Zell-Lymphom}

\section{A. Koch, P. Thomaschewski, U. Wollina}

Anamnese: Der 51-jährige Patient stellte sich erstmals im Januar 2014 zur Abklärung einer seit ca. einem Jahr bestehenden progredienten generalisierten ekzematoiden Hautveränderung mit begleitenden peripheren Lymphknotenschwellungen vor.

Allgemeinbefund: Zum Aufnahmezeitpunkt gab der Patient eine leicht reduzierte Allgemeinsymptomatik mit Abgeschlagenheit und Müdigkeit an. Er klagte noch über leichte Rückenbeschwerden. Deutliche Lymphknotenschwellungen axillär und inguinal beidseits waren nachweisbar. Im Verlauf der Erkrankung verschlechterte sich der Allgemeinbefund dramatisch mit ausgeprägter B-Symptomatik und Gewichtsverlust.

Hautbefund: Bei der klinischen Untersuchung fiel eine generalisierte erythrodermatische, infiltrierte, schuppende Haut auf $(\bullet$ Abb.3). Palmoplantar fanden sich gelbliche Hyperkeratosen. Im Krankheitsverlauf entwickelten sich schmerzhafte subkutane Infiltrate über der rechten Beckenschaufel.

Laborbefunde: Siehe $\bullet$ Tab. 1.

Bildgebende Diagnostik: Im CT unauffällige mediastinale und hiläre Lymphknoten. Deutlicher Nachweis von vergrößerten Lymphknoten axillär und inguinal. Retroperitoneal und mesenterial zahlreiche bis $2 \mathrm{~cm}$ große Lymphknoten. Deutlicher Nachweis inguinaler und iliakaler Lymphknoten. Im Verlauf deutlich größenprogrediente diffuse Lymphominfiltration des rechtsseitigen M. iliacus inklusive ossärer Arrosion der rechten Beckenschaufel.

Histologie: In der Hautbiospie geringer Infiltratepidermotropismus. Dichtes, gut abgrenzbares Lymphoidzellinfiltrat. Nachweis von Pautrierschen Mikroabszessen. Die Lymphoidzellen erweisen sich fast ausschließlich als T-Zell-reaktiv mit deutlichem Überwiegen von CD4-positiven T-Helfer-Zellen. Dermale und intradermale Ansammlung CD30-positiver blastärer Lymphoidzellen. Im Verlauf Transformation des T-Zell-Lymphoms in eine großzellige Variante eines CD30-positiven T-Zell-Lymphoms. ALK-Kinase negativ ( $\bullet$ Abb.4a und b).

Nahezu vollständig aufgehobene Lymphknotenstruktur mit einem dichten monomorphen Infiltrat kleiner lymphoider Zellen 


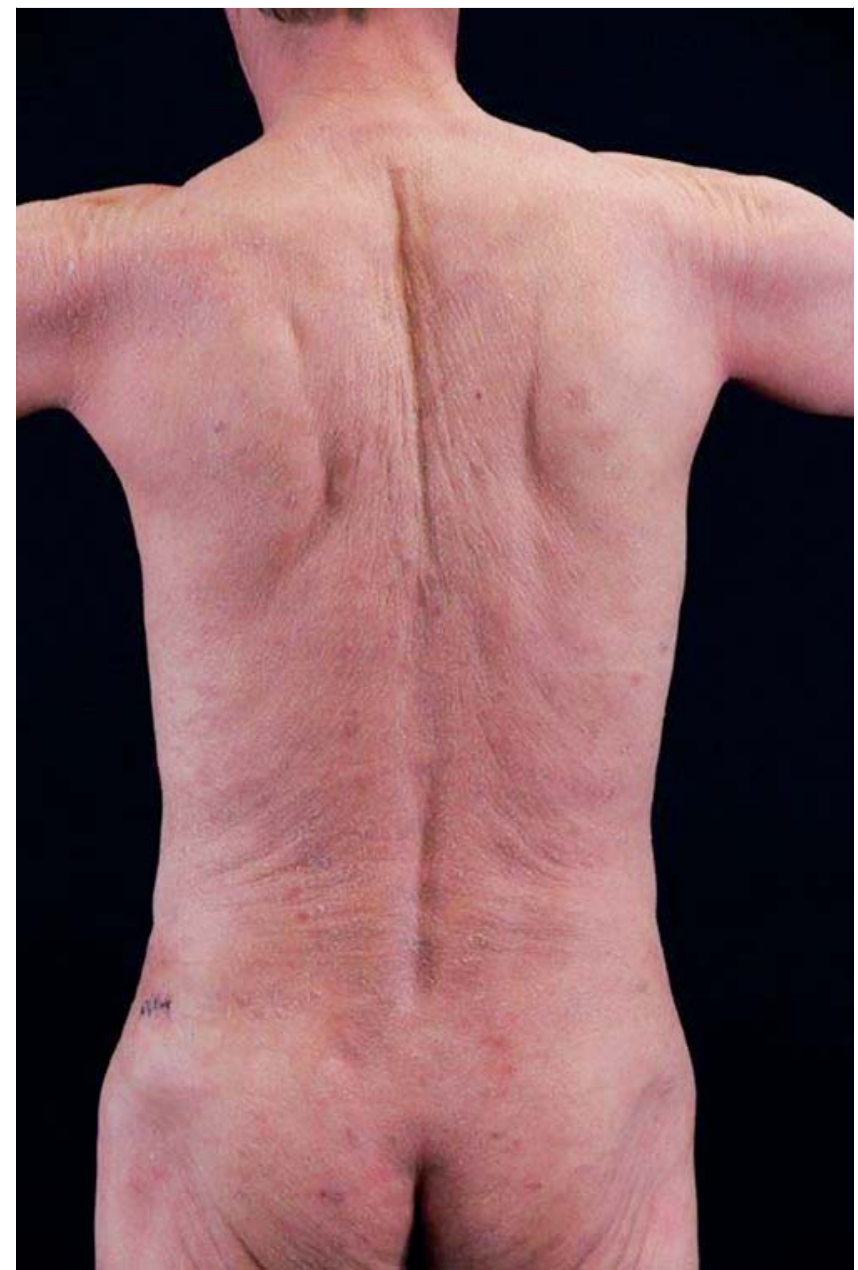

Abb.3 Erythrodermie bei großzellig anaplastischem CD30-positivem T-Zell-Lymphom.

Tab. 1 Laborbefunde.

\begin{tabular}{|llll|}
\hline & bei Aufnahme & im Verlauf & Normbereich \\
\hline Leukozyten & 29,1 & 12,43 & $3,8-11,0 \mathrm{Gpt} / \mathrm{I}$ \\
\hline Lymphozyten & 12,4 & 12,0 & $1,0-4,0 \mathrm{Gpt} / \mathrm{l}$ \\
\hline LDH & 10,5 & 4,16 & $2,25-3,75 \mu \mathrm{kat} / \mathrm{I}$ \\
\hline Sézaryzellen & 40 & 0 & $0 \%$ \\
\hline Thymidinkinase & 69 & n.d. & $0-6,1 \mathrm{U} / \mathrm{I}$ \\
\hline IL-2-Rezeptor & 3489 & n.d. & $223-710 \mathrm{U} / \mathrm{ml}$ \\
\hline CD4/CD8-Ratio & 8,64 & n.d. & $1,0-2,3$ \\
\hline
\end{tabular}

mit stark positiver Reaktion für CD3, CD4 und CD5. Eingestreut betont $\mathrm{CD} 30$-positive blastäre Zellen, wobei keine Hodgkin-Morphologie erkennbar ist.

Therapie und Verlauf: Mit dem Nachweis eines T-Zell-Lymphoms unter dem Bild einer Mycosis fungoides mit hämatogener Beteiligung (Sézary-Syndrom) anhand von Haut- und Lymphknotenhistologie sowie laborchemischen Daten erfolgte die Therapieeinleitung mit Methotrexat (MTX) $20 \mathrm{mg} /$ Woche. Zusätzlich wurden eine Bade-PUVA-Therapie sowie eine Lokaltherapie mit steroidhaltigen Externa durchgeführt. Im Juni 2014 erfolgte eine Therapieumstellung auf Bexaroten-Kapseln $75 \mathrm{mg}$ (2-2-2). Bei weiterer klinischer Verschlechterung des Befundes mit Knochenmarkbeteiligung wurde im Oktober 2014 eine weitere Therapieeskalation mit Durchführung von vier Zyklen einer modifizierten

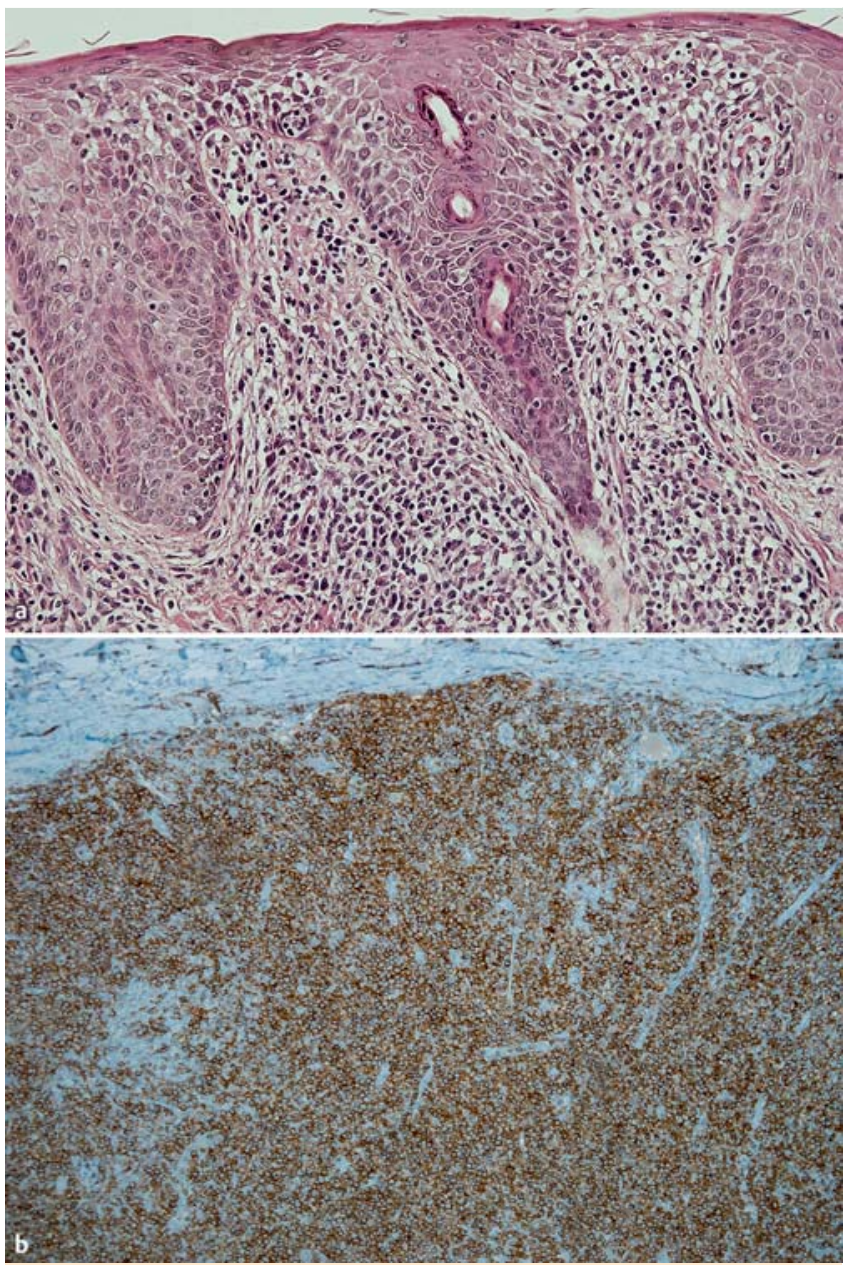

Abb.4 Histologie des großzelligen anaplastischen CD30-positiven T-ZellLymphoms. a Hämatoxylin-Eosin (×20). b CD4-Immunperoxidasefärbung $(\times 20)$.

CHOP-Chemotherapie (Cyclophosphamid, Doxorubicin) begonnen. Ein kurz vor der Ulzeration stehendes inguinales Lymphompaket rechtsseitig wurde strahlentherapeutisch behandelt. Aufgrund weiterer Verschlechterung des Allgemeinzustandes mit Gewichtsabnahme und starken Schmerzen im Bereich der rechtsseitigen Beckenschaufel infolge Lymphominfiltration in Knochen, M. sartorius und M. iliacus stellten wir den Patienten unseren Onkologen vor. Diese entschieden sich im Februar 2015 aufgrund eines Gestaltswandels des Lymphoms mit Übergang in ein großzelliges, anaplastisches CD30-positives Lymphom zur Einleitung einer Immunchemotherapie mit Brentuximab-Vedotin (Adcetris $^{\mathrm{R}}$ ). Alle drei Wochen erfolgt eine Infusion, die bislang sehr gut vertragen wurde, und zu einer dramatischen Besserung des Allgemeinbefindens und einem Rückgang des T-Zell-Lymphoms führte.

Kommentar: Lymphome stellen eine vielgestaltige Krankheitsgruppe dar und lassen sich nicht immer gleich korrekt einordnen. Aufgrund des klinischen Bildes, der Histologie und der Laborparameter mit Nachweis von Sézaryzellen im Blut wurde zunächst die Diagnose eines Sézary-Syndroms gestellt. In der Folge zeigte sich ein Übergang in ein CD30-positives anaplastisches großzelliges T-Zell-Lymphom. Eine großzellige Transformation bei der Mycosis fungoides (Sézary-Syndrom) ist relativ selten und mit einem aggressiveren klinischen Verlauf assoziiert [1]. 
Das CD30-positive anaplastische großzellige T-Zell-Lymphom (ALCL) stellt eine seltene Lymphomvariante dar. Man unterscheidet hier drei Unterformen: das primär systemische ALK-positive ALCL (ALKom), das primär systemische ALK-negative ALCL und das primär kutane ALCL [2]. Das anaplastische großzellige Lymphom wurde erstmals 1985 beschrieben und 1994 als eigenständige Entität in die REAL-Klasssifikation aufgenommen [3]. Der immunhistochemische Nachweis der ALK-Kinase in den Lymphomzellen ist von großer prognostischer Bedeutung, da er mit einer signifikant besseren Prognose einhergeht [4]. Während das ereignisfreie Überleben nach fünf Jahren bei ALK-positiven ALCL bei $60 \%$ liegt, beträgt es bei ALK-negativen ALCL nur 36\%. Das Gesamtüberleben nach fünf Jahren ist bei den ALKomen mit 70\% deutlich besser als bei den ALK-negativen ALCL mit 49\%. ALK-positive ALCL treten ganz überwiegend bei jungen männlichen Patienten auf, während ALK-negative ALCL bei älteren Patienten auftreten und keine Geschlechterbevorzugung aufweisen.

Brentuximab-Vedotin gehört zu den Antikörper-Drug-Konjugaten. Es bindet an CD30-exprimierende Zellen, schleust ein an den Antikörper gekoppeltes Zytostatikum (Monomethyl-Auristatin E) zielgerichtet in die Zelle ein und bedingt dadurch den Zelltod [5]. Genutzt werden kann dieses Medikament sowohl bei therapierefraktären Hodgkin-Lymphomen als auch beim CD30positiven anaplastischen großzelligen T-Zell-Lymphom. Brentuximab-Vedotin zeigt ein hohes Ansprechen bei moderatem Nebenwirkungsprofil. Am häufigsten kommt es zu einer Neuropathie, Leukopenie, Thrombopenie, Übelkeit und Abgeschlagenheit. Brentuximab-Vedotin stellt somit ein neues innovatives Medikament für CD30-positive Lymphome dar und bietet den Patienten neben einem geringen Nebenwirkungsprofil ein hohes Maß an Lebensqualität [5].

\section{Literatur}

1 Diamandidou E, Colome-Grimmer M, Fayad L et al. Transformation of mycosis fungoides/Sezary syndrome: clinical characteristics and prognosis. Blood 1998; 92: 1150

2 Drexler HG, Gignac SM, von Wasielewski R et al. Pathobiology of NPMALK and variant fusion genes in anaplastic large cell lymphoma and other lymphomas. Leukemia 2000; 14: 1533 -1559

3 Stein H, Mason DY, Gerdes J et al. The expression of the Hodgkin's disease associated antigen $\mathrm{Ki}-1$ in reactive and neoplastic lymphoid tissue: evidence that Reed-Sternberg cells and histiocytic malignancies are derived from activated lymphoid cells. Blood 1985; 66: 848-858

4 Falini B, Pileri S, Zinzani PL et al. ALK+ lymphoma: clinico-pathological findings and outcome. Blood 1999; 93: 2697-2706

5 Rothe A, Sasse S, Goergen $\mathrm{H}$ et al. Brentuximab vedotin for relapsed or refractory $\mathrm{CD} 30+$ hematologic malignancies: the German Hodgkin Study Group experience. Blood 2012; 120: 1470-1472

\section{Kutane leukozytoklastische Vaskulitis unter dem klinischen Bild einer Panarteriitis nodosa cutanea benigna}

\section{Krönert, K. Hasenöhrl, J. Schönlebe}

Anamnese: Im Oktober 2013 bemerkte der 75-jährige Patient ein Malum perforans an der linken Großzehe. Eine Polyneuropathie unklarer Ätiologie bestand bereits seit ca. 1994. Kurze Zeit später kam es zu einer lokalen eitrigen Entzündungsreaktion mit Rötung und Schwellung der linken Großzehe, die mit Levofloxacin behandelt wurde. Etwa zwei Monate später zeigten sich an beiden Unterschenkeln Erytheme und an beiden Oberschenkeln

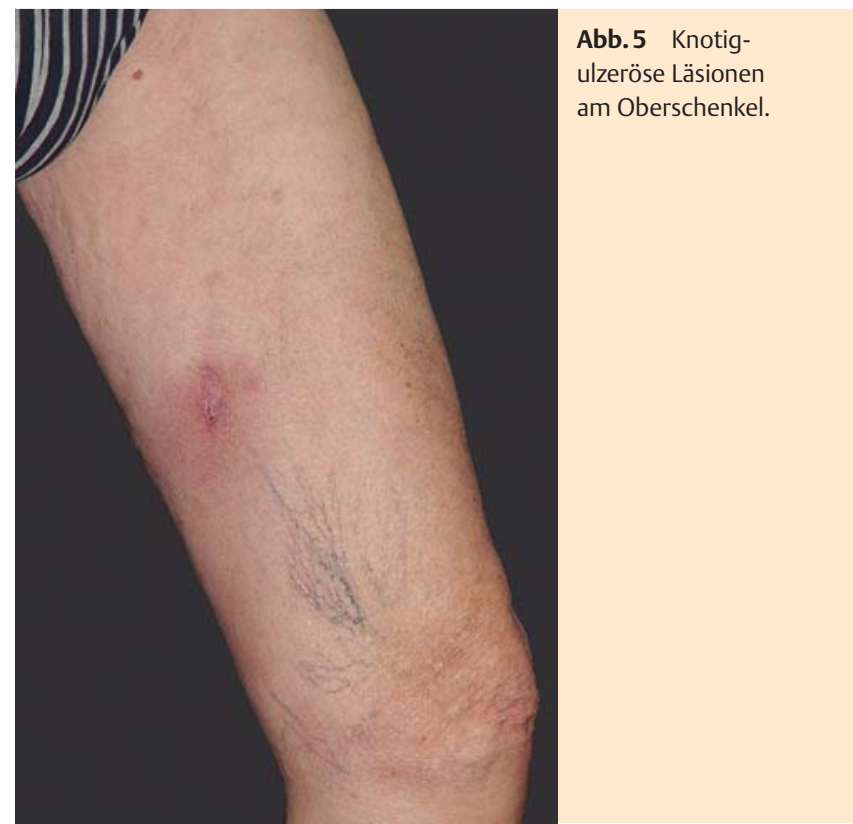

druckschmerzhafte Knötchen. Das Allgemeinbefinden war unverändert gut. Es bestanden weder grippeähnliche Symptome noch eine B-Symptomatik. Es ließen sich keine weiteren Infekte im Vorfeld eruieren. Unter chronischen Gelenkbeschwerden v.a. an beiden Händen, die als Polyarthrose mit Pfropfarthritis diagnostiziert wurden und mit MTX plus Prednisolon therapiert werden, leidet der Patient bereits seit 2000. 1994 wurde zudem ein Rektumkarzinom vollständig entfernt. Ein Diabetes mellitus Typ 2 besteht seit 2001. Ferner sind eine absolute Arrhythmie bei Vorhofflimmern sowie eine arterielle Hypertonie bekannt.

Allgemeinbefund: Auffällig waren Heberdenknoten an den Fingern, ein nicht vollständiger Faustschluss beider Hände, druckschmerzhafte, aber nicht entzündlich geschwollene Fingergelenke. Zusätzlich zeigten sich eine eingeschränkte Beweglichkeit im Bereich der LWS sowie arrhythmische Herztöne.

Hautbefund: An beiden Unterschenkeln sahen wir eine ödematöse Schwellung mit infiltrativen, teils petechialen, lividroten Erythemen. Zudem fanden sich druckschmerzhafte, zum Teil strangförmig angeordnete subkutane Knötchen mit lividroter Umgebung an beiden Oberschenkeln ( $\bullet$ Abb.5).

Histologie (HE, Giemsa, PAS, Eisenreaktion): Oberflächliche Dermis ödematös aufgelockert. Insgesamt schütteres, oberflächlich und tief perivaskuläres, lymphomonozytoides, entzündliches Infiltrat mit wenigen beigemischten Mastzellen und neutrophilen Granulozyten. Im Interstitium diskrete diffuse Vermehrung fibrohistiozytärer Zellen. In der Subkutis oberflächlich ein streifiges, dichtes, perivaskulär akzentuiertes, gemischtzelliges, entzündliches Infiltrat mit randlich großleibigen Zellen offenbar histiozytären Ursprungs, entzündlicher Infiltration von Perineuralscheiden und Anschnitten mehrerer Blutgefäße (offenbar postkapilläre Venolen) mit thrombotischen Gefäßlichtungsverschlüssen, Gefäßwandnekrosen und granulozytär entzündlicher Gefäßwandinfiltration mit wechselnd stark ausgeprägter perivaskulär interstitieller Leukozytoklasie ( $\bullet$ Abb.6). Keine Infiltrateosinophilie. Eisenreaktion negativ. Direkte Immunfluoreszenzuntersuchung: Kein Nachweis spezifischer Immunreaktionen für IgA, IgM, IgG, Fibrin und C3.

Laborbefunde: Das CRP war mit $16 \mathrm{mg} / \mathrm{l}$ nur leicht erhöht. Unauffällig bzw. negativ waren ANA, ANCA, AMA, Komplement, Kryo- 


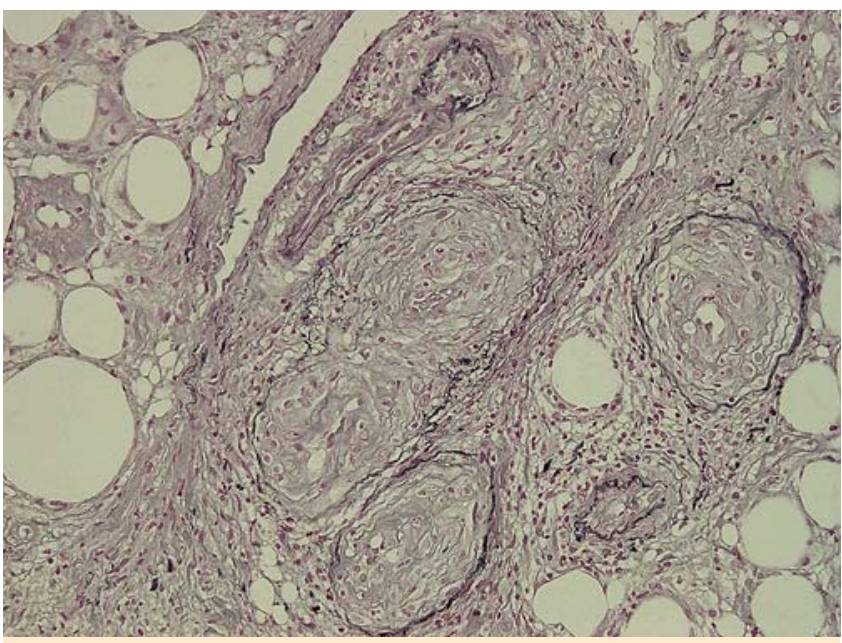

Abb. 6 Histologie (Hämatoxylin-Eosin×20).

globuline, Cardiolipin-AK, IgA, Immunfixation, Differenzialblutbild, BSG, RF, CCP, Hepatitisserologie, HIV-Test und TPHA.

Bildgebende Diagnostik: Schwere Arthrose der DIP-Gelenke des dritten bis fünften Strahles rechts sowie der PIP-Gelenke 5 beidseits, geringer auch der übrigen PIP-Gelenke. Ausgeprägte arthrotische Gelenkspaltverschmälerung der MCP-Gelenke mit hakenförmiger Deformität des Köpfchens von Os metakarpale V beidseits. Schwere Rhizarthrosis deformans mit heterotopen Ossifikationen.

Therapie und Verlauf: Wir erhöhten die interne Prednisolondosis auf $30 \mathrm{mg} / \mathrm{d}$ und therapierten lokal mit steroidhaltigen Externa und Kompressionsbandagierung. Unterstützend erfolgte eine physiotherapeutische Krankengymnastik. MTX wurde bis zum Abschluss der Diagnostik pausiert, später ambulant wieder auf $15 \mathrm{mg} /$ Woche eingestellt und das Prednisolon auf eine Erhaltungsdosis von $2,5 \mathrm{mg} / \mathrm{d}$ abgesenkt. Darunter kam es langsam zur Abheilung der Rötungen und zum Rückgang der knotigen Veränderungen. Es traten keine neuen entzündlichen Hautveränderungen auf, Restzustände im Sinne von hyperpigmentierten Arealen an Ober- und Unterschenkel bestehen noch fort.

Kommentar: Vaskulitiden sind stets eine Herausforderung. Die Diagnosestellung ist aufgrund des breiten Ursachenspektrums, der variablen klinischen Symptomatik und der möglichen Organbeteiligung aufwändig und fordert oft die Zusammenarbeit mehrerer Fachgebiete. Erst nach eingehender Anamnese, richtungsweisender Labor- und Organdiagnostik sowie histologischer Beurteilung einer Gewebeprobe kann die entsprechende Diagnose gesichert und die erforderliche Therapie gebahnt werden. Wichtig ist der Ausschluss einer systemischen Vaskulitis [1].

Unser Patient stellte sich mit unklaren petechialen Rötungen an beiden Unterschenkeln (ohne Papeln) und knotigen, zum Teil strangförmig angeordneten entzündlichen Hautveränderungen an beiden Oberschenkeln vor. Diese klinische Befundkonstellation ließ uns nicht in erster Linie an eine leukozytoklastische Vaskulitis denken, sondern eher eine Panarteriitis nodosa differenzialdiagnostisch in Erwägung ziehen. Aber histologisch ergab sich eindeutig eine entzündliche Veränderung kleiner Gefäße mit Leukozytoklasie ohne IgA-Ablagerungen. Parallel erfolgte eine umfangreiche Diagnostik zum Ausschluss einer systemischen Vaskulitis, einer Tumorerkrankung, chronischer Infektionserkrankungen und einer floriden destruierenden rheumatoiden Erkrankung. Als mögliche Ursachen der nun diagnostizierten kutanen leukozytoklastischen Vaskulitis, auch als Hypersensitivitätsangiitis bezeichnet, kommen sowohl die stattgehabte eitrige Entzündung des Druckulkus an der linken Großzehe als auch deren antibiotische medikamentöse Behandlung in Betracht. Alle Hauterscheinungen heilten rasch unter einer kurzfristigen Erhöhung der internen Prednisolondosis ab und rezidivierten auch nicht.

Die kutane leukozytoklastische Angiitis/Vaskulitis ohne Systemerkrankung findet sich in der aktuellen Nomenklatur der Chapel-Hill-Konsensuskonferenz von 2012 in der Gruppe der Einzelorganvaskulitis (SOV) und ist als Ausschlussdiagnose zu betrachten [2].

\section{Literatur}

1 Wollina U, Unger L, Haroske $G$ et al. Classification of vascular disorders in the skin and selected data on new evaluation and treatment. Dermatol Ther 2012; 25: 287-296

2 Jennette JC, Falk RJ, Bacon PA et al. 2012 revised international Chapel Hill consensus conference nomenclature of vasculitides. Arthritis Rheum 2010; 65: 1-11

\section{Erythema gyratum repens bei Ösophagus-Karzinom \\ $\nabla$}

\section{Langner, S. Schamfuß}

Anamnese: Der 76-jährige Patient stellte sich mit seit Oktober 2013 überwiegend am Körperstamm lokalisierten, juckenden und progredienten Hautveränderungen vor. Es bestand der Verdacht einer hypereosinophilen Dermatitis und differenzialdiagnostisch auch einer systemischen Mastozytose bei ambulant laborchemisch auffälliger Hypereosinophilie. Vor ca. 6 Jahren hätten bereits ähnliche Hautveränderungen bestanden, welche unter UV- und Lokaltherapie abgeheilt wären. Bereits im April 2014 war der Patient unter dem Verdacht einer Urtikaria pigmentosa mittels Bade-PUVA behandelt worden.

Anamnestisch sei es jedoch in den letzten Wochen zu einer akuten Exazerbation gekommen, verbunden mit Appetitlosigkeit, Abgeschlagenheit, massivem Juckreiz und Gewichtsverlust. Hautbefund: Es imponierten am Körperstamm und an den proximalen Extremitätenabschnitten multiple, hochrote Papeln und Plaques. Das Darier-Zeichen war negativ. Im stationären Verlauf entwickelten sich bevorzugt an den unteren Extremitäten kokar-

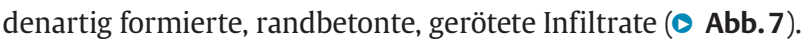

Histologie der Haut (HE, Eisenreaktion): Epidermis mit orthokeratotischer Verhornung mit Serumeinschlüssen und Einschlüssen von Leukozytenzelltrümmern. In zentralen Präparateabschnitten fanden sich in unterschiedlichem Maße mäßig starke, teils uncharakteristisch, teils angedeutet psoriasisform hyperplastisch mit geringgradiger epidermaler Spongiose sowie geringgradiger flächiger Hyperparakeratose $(\bullet$ Abb.8).

In Epidermis und oberem Korium ein mitteldichtes, teils perivaskulär, teils interstitiell lokalisiertes lymphomonozytoides, entzündungszelliges Infiltrat mit Beimengungen neutrophiler und eosinophiler Granulozyten in wechselnder Zahl. Gering vermehrte Mastzellen. Keine zellulären Atypien. Kein Pilznachweis.

Histologie Ösophagus: Mäßig differenziertes Adenokarzinom auf dem Boden einer Barrett-Schleimhaut.

Laborbefunde: Pathologisch waren Hämoglobin $8,2 \mathrm{mmol} / \mathrm{l}$, Hämatokrit 0,4l/1, Neutrophile 8,90 Gpt/l, Eosinophile 0,70 Gpt/l, und Mastzelltryptase $22,1 \mu \mathrm{g} / \mathrm{l}$.

Bildgebende Diagnostik: Keine Hinweise auf Metastasierung. 


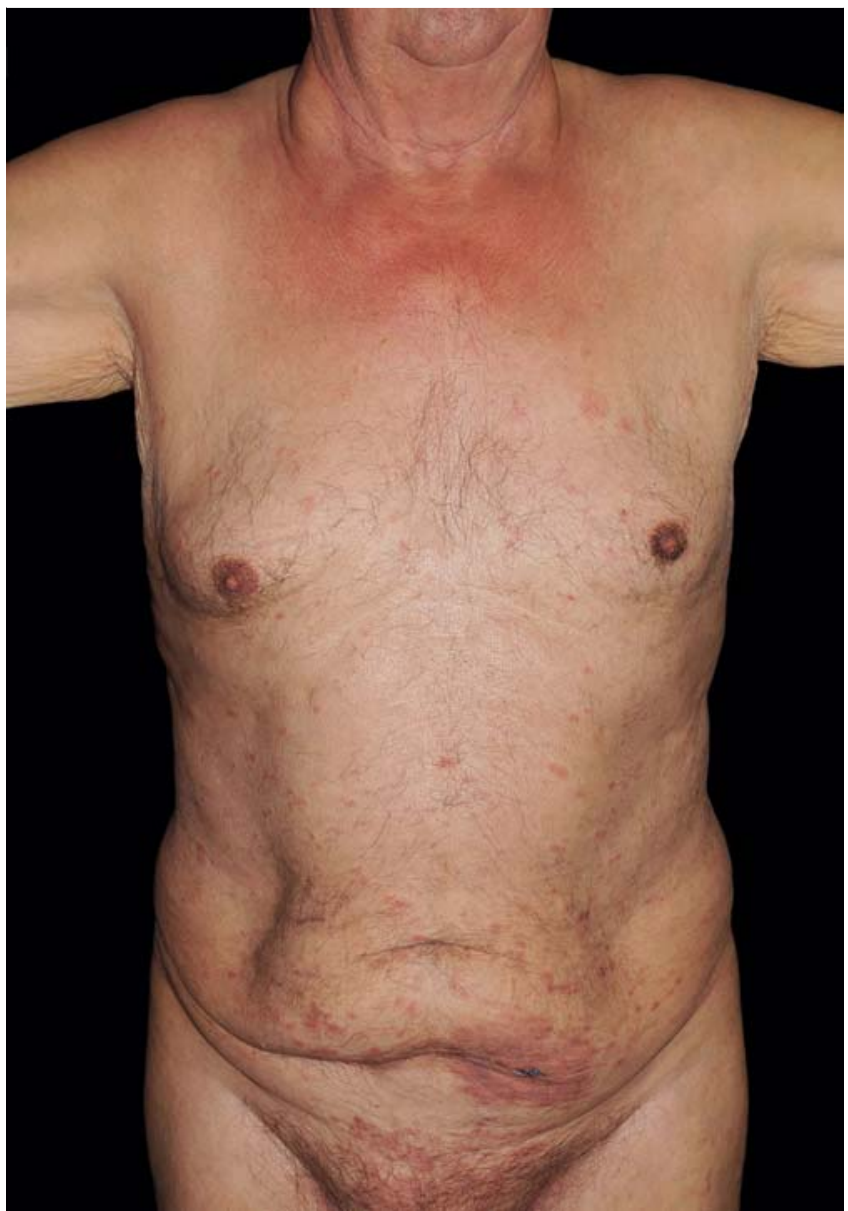

Abb.7 Klinischer Befund.

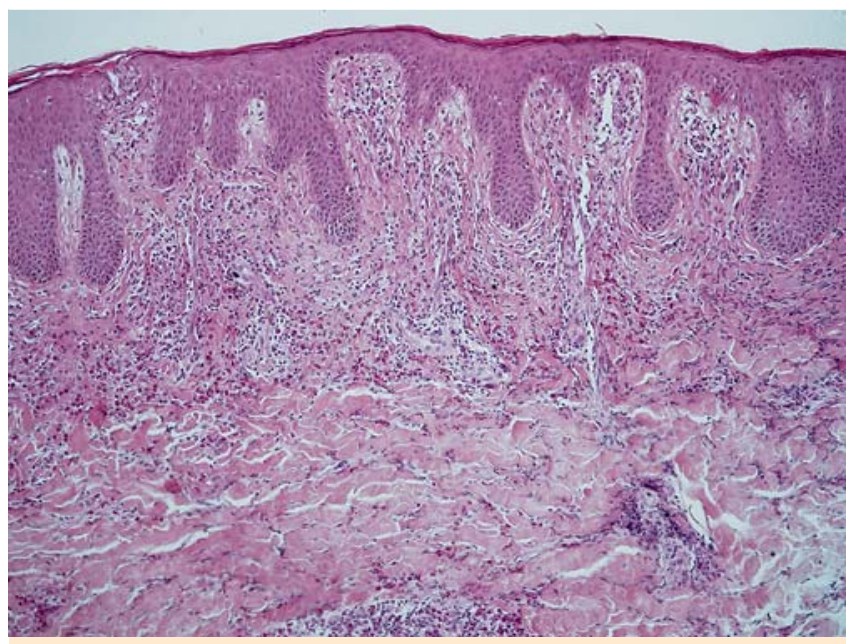

Abb.8 Hämatoxylin-Eosin (×10).

Therapie und Verlauf: Wir behandelten die Körperhaut mit Fluocinolonacetonid (Jellinsalbe) $2 \times$ tägl. und führten zusätzlich eine Bade-PUVA über insgesamt 8 Sitzungen durch. Gegen den starken Juckreiz ordinierten wir Fexofenadin und Ranitidin.

Der initiale Verdacht einer systemischen Mastozytose bestätigte sich nicht. In der Gastroskopie ergaben sich auffällige Schleimhautveränderungen am distalen Ösophagus, welche sich histologisch als mäßig differenziertes Adenokarzinom auf dem Boden einer Barrett-Schleimhaut darstellten. Dieser Tumor wurde strahlentherapeutisch behandelt.

Kommentar: Die Erstbeschreibung des Erythema gyratum repens erfolgte durch Gammell im Jahre 1952 bei einer 55-jährigen Frau mit metastasierendem Adenokarzinom der Mamma. Bereits 2 Tage nach der Mastektomie und Entfernung der axillären Lymphknoten blassten die Erytheme ab und nach 6 Wochen waren sie vollständig verschwunden [1].

Ein Erythema gyratum repens tritt in der überwiegenden Anzahl der publizierten Fälle mit einem malignen Tumor assoziiert auf. Der häufigste maligne Tumor ist mit 32\% das Bronchialkarzinom, gefolgt vom Ösophagus-Karzinom (8\%) und dem Mamma-Karzinom (6\%). Pathophysiologisch wird dabei eine tumorinduzierte humorale und/oder zelluläre Immunantwort vermutet, die sich dann im Sinne einer Kreuzreaktion auch an der Haut manifestiert oder Ablagerungen von Tumorantigen-Antikörper-Immunkomplexen an der Basalmembran verursacht [2].

Klinisch imponieren anuläre, girlandenförmige oder spiralig verschlungene, randbetonte, meist parallel verlaufende, leicht infiltrierte Läsionen und schuppende Erytheme. Die Ausbreitungsgeschwindigkeit beträgt bis zu einem Zentimeter pro Tag.

In $2 / 3$ der publizierten Fallbeschreibungen konnte ein maligner Tumor nachgewiesen werden, in dem restlichen Drittel der Fälle zeigte sich eine überwiegende Assoziation mit weiteren dermatologischen Erkrankungen [3]. Somit stellt das Erythema gyratum repens nicht ausschließlich eine obligate Paraneoplasie dar.

\section{Literatur}

1 Gammel JA. Erythema gyratum repens; skin manifestations in patient with carcinoma of breast. AMA Arch Derm Syphilol 1952; 66: 494505

2 Silva JA, Mesquita Kde C, Igreja AC et al. Paraneoplastic cutaneous manifestations: concepts and updates. An Bras Dermatol 2013; 88: 9-22

3 Rongioletti F, Fausti V, Parodi A. Erythema gyratum repens is not an obligate paraneoplastic disease, a systemic review of the literature and personal experience. J Eur Acad Dermatol Venerol 2014; 28: 112 -115

\section{Idiopathisches Kaposi-Sarkom}

\section{$\nabla$}

\section{Klingner, S. Schmidt, J. Schönlebe}

Anamnese: Der 61-jährige Patient berichtete über multiple, rote bis bräunliche Hautveränderungen, welche seit Mitte 2013 erstmals am linken Unterschenkel aufgetreten sind. In der Folge entstanden rasch neue Herde an den Extremitäten. Eine B-Symptomatik mit Gewichtsverlust, Nachtschweiß, Fieber oder Appetitlosigkeit wurde verneint.

Allgemeinbefund: Wir sahen einen schlanken Patienten in gutem Allgemeinzustand. Inguinal beidseits ließen sich gut verschiebliche, ovaläre Lymphknoten palpieren.

Hautbefund: An beiden Unterschenkeln, am rechten Handgelenk und Unterarm sowie am linken Oberschenkel medial fielen mehrere papulöse und teils knotige, rote bis bräunliche Herde auf (๑ Abb.9).

Histologie: In mehreren Hautexzisaten zeigte sich ein ähnliches Bild. Die Epidermis ist verschmälert und hyperkeratotisch. Korial fällt ein zellreicher faszikulär-spindelzelliger Tumor mit ovalären Kernen, allenfalls geringer Kernatypie, mäßig vielen Mitosen, einzelnen PAS-positiven, kleinen, zytoplasmatischen Globuli sowie verschiedentlich schlitzförmigen, mit Erythrozyten ausgefüllten Hohlräumen auf. Benachbart finden sich eine mäßige 


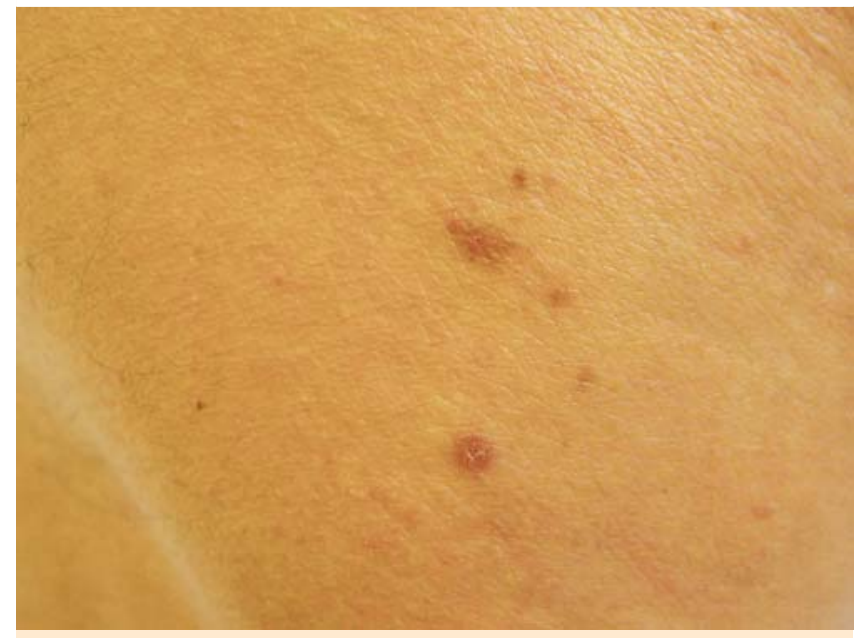

Abb.9 Klinisches Bild des klassischen Kaposi-Sarkoms.

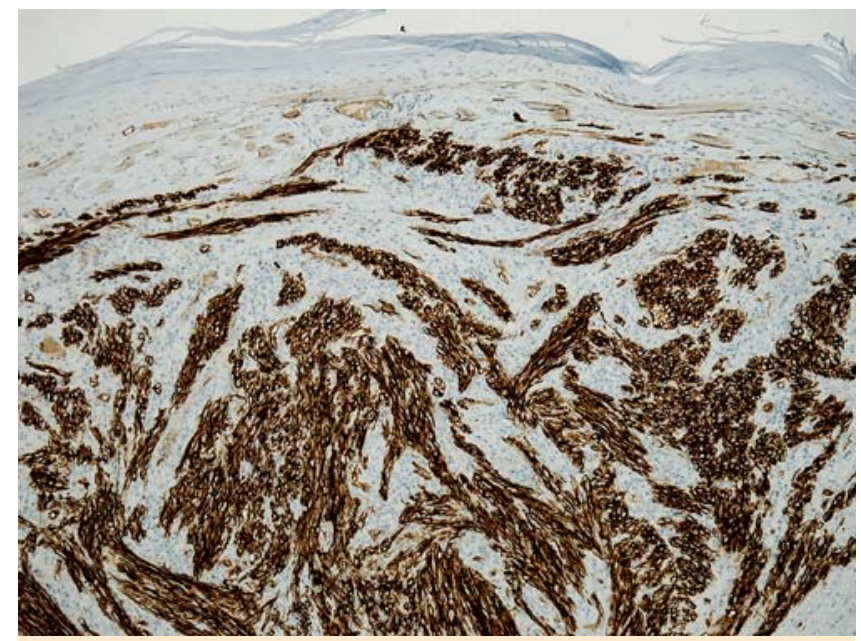

Abb. 10 CD34-positives Kaposi-Sarkom $(\times 10)$.

lymphoplasmazelluläre Entzündungsreaktion und betonte Kapillarektasien. Immunhistochemisch zeigen die Tumorzellen eine positive Reaktion für CD31 bzw. CD34 sowie gegenüber dem HHV8-Antikörper ( $\bullet$ Abb. 10).

Laborbefunde: Pathologisch waren die Erythrozyten mit 4,31 Tpt/l, die neutrophilen Granulozyten mit 1,5Gpt/l sowie das Hämoglobin mit $8,3 \mathrm{mmol} / \mathrm{l}$. Die Infektionsserologie (HIV, Lues) war negativ.

Therapie und Verlauf: Die Histologie ergab ein Kaposi-Sarkom. Da keine HIV-Infektion oder andere Immunsuppression besteht, liegt bei dem Patienten ein idiopathisches oder klassisches Kaposi-Sarkom ohne systemische Beteiligung vor. Alle Läsionen wurden im Gesunden entfernt und der Patient befindet sich in regelmäßiger hautärztlicher Kontrolle.

Kommentar: Das Kaposi-Sarkom (KS) gilt als eine multilokuläre maligne Neoplasie, ausgehend von den endothelialen Zellen. Man teilt klinisch-pathogenetisch in vier Gruppen ein: 1. das klassische Kaposi-Sarkom (gehäuft in Ost- und Südeuropa bei älteren Männern), 2. das iatrogene Kaposi-Sarkom bei Immunsuppression z. B. nach Organtransplantation, 3. das oft fulminant aggressive lymphadenopathische (endemische) Kaposi-Sarkom bei jungen Afrikanern und 4. das epidemische HIV-assoziierte Kaposi-Sarkom [1]. Dem KS geht eine Entzündungsreaktion voraus, die auf einer Infektion mit dem humanen Herpesvirus
Typ 8 (HHV-8) beruht [2]. Das Virus blockiert unter anderem Tumorsuppressorgene und hemmt die Proliferationsblockade der Endothelzellen, sodass eine ungehemmte Endothelproliferation in Gang gesetzt wird. Klinisch fällt das KS an der Haut wie bei unserem Patienten mit roten bis lividen oder bräunlichen Makeln und Plaques auf [1]. Die Prognose des Tumors ist vom Ausmaß des Haut- bzw. Organbefalls abhängig. Einzelne Herde sollten exzidiert oder einer Strahlentherapie zugeführt werden. Bei systemischem Befall muss eine Chemotherapie, z. B. mit liposomalem Doxorubicin erwogen werden. In letzter Zeit wurde Rapamycin, ein Hemmer des mTOR-Signalwegs, erfolgreich eingesetzt [3]. In unserem Krankengut zählt das klassische KaposiSarkom zu den seltenen Sarkom-Entitäten [4].

\section{Literatur}

1 Antman K, Chang Y. Kaposi's sarcoma. N Engl J Med 2000; 342: 1027 1038

2 Lebbé C. Humanes Herpesvirus 8 (HHV-8) und Kaposi-Sarkom. Hautarzt 2008; 59: $18-25$

3 Díaz-Ley B, Grillo E, Ríos-Buceta L et al. Classic Kaposi's sarcoma treated with topical rapamycin. Dermatol Ther 2015; 28: 40-43

4 Wollina U, Koch A, Hansel G et al. A 10-year analysis of cutaneous mesenchymal tumors (sarcomas and related entities) in a skin cancer center. Int J Dermatol 2013; 52: 1189-1197

\section{Basalzellkarzinom auf Radioderm $\nabla$ \\ C. Krönert, G. Scheibner}

Anamnese: Vor 40 Jahren wurde bei dem 74-jährigen Patienten an der Glabella ein Basalzellkarzinom bestrahlt. Etwa 38 Jahre darauf zeigte sich eine nicht heilende Erosion mit Krustenbildung. Eine ambulante Hautprobe ergab erneut ein Basalzellkarzinom.

Hautbefund: Über der linken Augenbraue fand sich eine ca. $15 \mathrm{~mm}$ große, atrophe, depigmentierte Narbe mit randbetont hyperpigmentiertem Saum und eine kleine postoperative Narbe nach Probeexzision ( $\bullet$ Abb. 11).

Histologie: Breitstreifige, auch in die basale mitresezierte mimische Muskulatur einstrahlende, zellarme, hyalinisierte, narbige Fibrose mit reaktiven Veränderungen der bedeckenden Epidermis sowie angrenzend breit bandförmiger Elastose, vereinbar

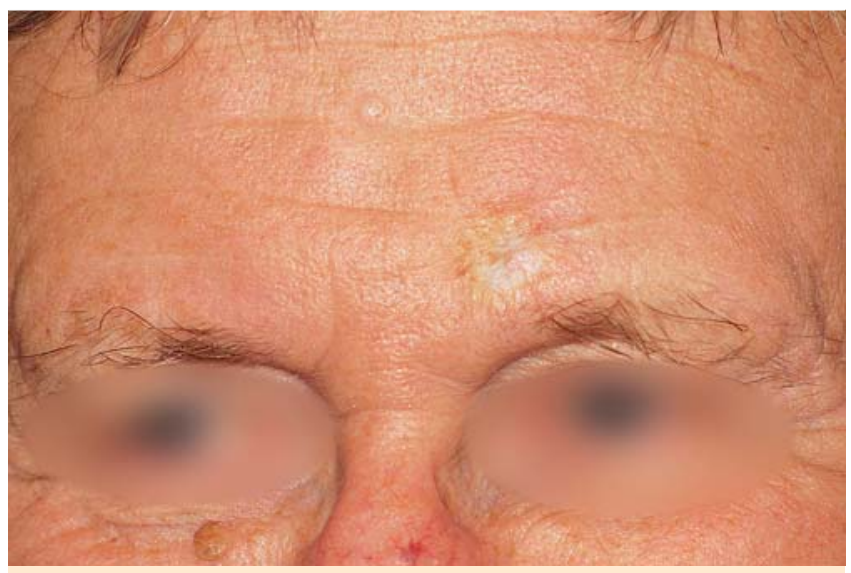

Abb.11 Basalzellkarzinom auf chronischem Radioderm. 
mit einem Radioderm, kein Anhalt für Basalzellkarzinomstrukturen, Narbenentfernung im Gesunden.

Therapie und Verlauf: Die Entfernung der Hautveränderung wurde im Rahmen eines zweizeitigen Vorgehens am 28.10.2014 in Lokalanästhesie unter einer Antibiotikaprophylaxe mit Cefuroxim $1000 \mathrm{mg}$ durchgeführt. Nach Eingang des histologischen Befundes und Nachweis eines Radioderms ohne weitere Basalzellkarzinomstrukturen wurde der Defekt am Folgetag mittels Dehnungslappenplastik verschlossen.

Kommentar: Vor 40 Jahren wurde bei unserem Patienten ein Basalzellkarzinom bestrahlt. Bestrahlungsmethoden unter Verwendung der Röntgenweichstrahlentherapie hatten seinerzeit vergleichbare Heilungsraten wie konventionelle operative Maßnahmen und waren zudem oft ästhetisch-funktionell ansprechend [1].

Heute wird gemäß der aktuellen S2-Leitlinie in erster Linie die komplette operative Entfernung des Tumors mittels mikrografischer Chirurgie („Mohs surgery“) empfohlen. Vorteilhaft sind die histologische Kontrolle und die zeitlich effektive Prozedur. Als nachteilig können sich Komplikationen wie Wundheilungsstörungen oder unschöne störende Narben erweisen [2].

Als alternatives Therapiekonzept wird heute nach wie vor die Bestrahlung des Basalzellkarzinoms durchgeführt. Indikationen zur Radiotherapie sind allgemeine oder lokale Inoperabilität, inkomplette chirurgische Entfernung (R1, R2) ohne Möglichkeit einer Nachresektion, zu erwartende funktionell einschränkende oder ästhetisch entstellende Ergebnisse oder eine fehlende OP-Einwilligung. Chronische Strahlenschäden treten bei verbesserten Dosierungskonzepten und dem Einsatz der Elektronenstrahlung von Linearbeschleunigern seltener auf [2].

Grenzstrahlung zeigt im Gegensatz zu anderen Strahlenqualitäten ein sehr geringes sekundäres Tumorrisiko $[3,4]$. Bekannt ist jedoch, dass in seltenen Fällen auf Strahlennarben Sekundärmalignome entstehen können. Dies sollte bei der Indikationsstellung zur Strahlentherapie - gerade bei jüngeren Patienten - berücksichtigt werden.

Ob es sich in unserem Fall um einen zufällig in dieser Lokalisation neu aufgetretenen Tumor oder um ein Rezidiv des vor 40 Jahren bestrahlten Basalzellkarzinoms handelt oder der Tumor eine Bestrahlungsfolge darstellt, kann nicht mit letzter Sicherheit geklärt werden.

Dieser Fall zeigt die Bedeutung von Aufklärung der Patienten über Therapierisiken und Tumorentstehung sowie die Bedeutung einer suffizienten Anleitung zur Selbstuntersuchung.

\section{Literatur}

1 Reymann F, Kopp $H$. Treatment of basal cell carcinoma of the skin with ultrasoft X-rays. Dermatologica 1978; 156: 40-47

2 Hauschild A, Breuninger $H$, Kaufmann $R$ et al. Brief S2k guidelines Basal cell carcinoma of the skin. J Dtsch Dermatol Ges 2013; 11 (Suppl 3): $10-5,11-6$

3 Lindelöf B, Eklund G. Incidence of malignant skin tumors in 14,140 patients after grenz-ray treatment for benign skin disorders. Arch Dermatol 1986; 122: $1391-1395$

4 Karagas MR, McDonald JA, Greenberg ER et al.; for the Skin Cancer Prevention Study Group. Risk of basal cell and squamous cell skin cancers after ionizing radiation therapy. J Natl Cancer Inst 1996; 88: $1848-$ 1853

\section{Pemphigus vulgaris als Zufallsbefund}

\section{$\nabla$}

\section{Langner, H. Mothes, G. Hansel}

Anamnese: Bei der 69-jährigen Patientin bestand an der linken Schläfe seit ca. 1 Jahr eine langsam größenprogrediente, rezidivierend nässende, blutende und erosive Hautveränderung. Unter dem Verdacht eines Basalzellkarzinoms wurde die Patientin zur operativen Sanierung aufgenommen.

Hautbefund: Klinisch bestand an der linken Schläfe eine $20 \times$ $15 \mathrm{~mm}$ große, hämorrhagische, krustös belegte und unscharf begrenzte Läsion.

Histologie von der linken Schläfe: Ausgedehnte suprabasale akantholytische Spalt- und beginnende Blasenbildungen mit Einblutungen in die Spalträume bzw. die Blasenlichtungen. Solare Elastose. Im Papillarkörper und oberflächlichen Korium bestehen überwiegend mitteldichte, perivaskulär lokalisierte, lymphomonozytoide Entzündungszellinfiltrate mit Beimengungen von eosinophilen Granulozyten und Plasmazellen. Verdacht auf Pemphigus vulgaris ( $\bullet$ Abb. 12).

Histologie von der Mundschleimhaut mit direkter Immunfluoreszenz: Die beschriebenen immunfluoreszenzoptischen Befunde stützen die im Vorbefund (von der Schläfe) geäußerte Verdachtsdiagnose eines Pemphigus vulgaris.

Laborbefunde: Pemphigus-Antikörper positiv mit $1: 160$.

Therapie und Verlauf: Die Exzision der Hautveränderung erfolgte in Lokalanästhesie mit anschließendem Defektverschluss mittels Vollhauttransplantat von links zervikal.

Nach Vorlage der überraschenden Histologie mit dem Verdacht eines Pemphigus vulgaris und auf genaue Nachfrage berichtete die Patientin zudem noch von rezidivierend auftretenden schmerzhaften Erosionen und Bläschen im Bereich der Mundschleimhaut. Bei genauer Inspektion konnte eine aphthöse Läsion an der Unterlippe festgestellt werden ( $\bullet$ Abb.13). Eine entnommene Biopsie aus dieser Läsion sowie die laborchemisch positiven Pemphigus-Antikörper bestätigten die Diagnose eines Pemphigus vulgaris.

Wir begannen eine Systemtherapie mit Prednisolon $50 \mathrm{mg}$ tägl. per os unter Magenschutz mit Pantoprazol $20 \mathrm{mg} / \mathrm{d}$. Die Prednisolondosis konnte im Verlauf auf aktuelle $10 \mathrm{mg} / \mathrm{d}$ reduziert werden.

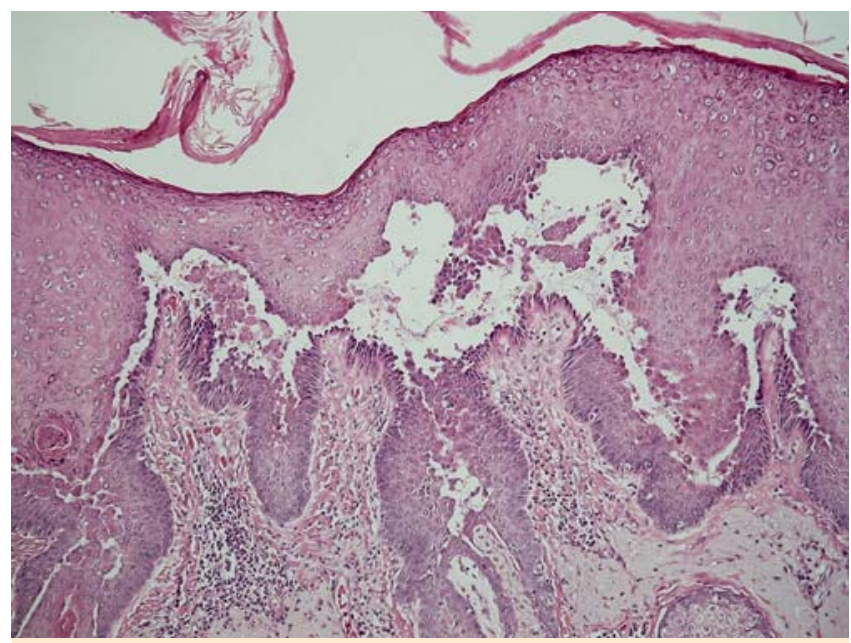

Abb.12 Histologie von der Schläfe (Hämatoxylon-Eosin × 10). 


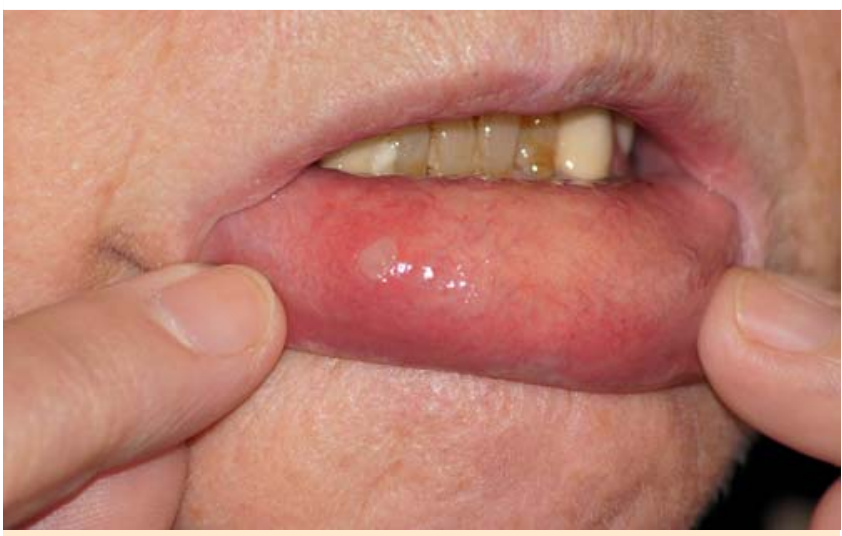

Abb.13 Aphthöse Lippenläsion bei Pemphigus vulgaris.

Kommentar: Der Pemphigus vulgaris, die häufigste PemphigusVariante, ist eine seltene, weltweit vorkommende bullöse Autoimmundermatose mit einem potenziell letalen Krankheitsverlauf. Die Inzidenz in Deutschland liegt geschätzt bei ca. 2 Neuerkrankungen pro Million Einwohner pro Jahr mit einer Mortaliät von $5-10 \%[1]$.

Wie unsere Beobachtung dokumentiert, stellt sich die Erkrankung klinisch jedoch nicht immer nach Lehrbuch dar. Klinisch untypische Verläufe lassen an zahlreiche Differenzialdiagnosen denken.

Die größte Herausforderung stellt nach wie vor die Behandlung dar. Vor Einführung der Glukokortikoide verstarben etwa $75 \%$ aller Patienten innerhalb eines Jahres nach Diagnosestellung vor allem an Sekundärkomplikationen wie ausgedehnten Infektio- nen oder Sepsis [2]. Mit der Anwendung von Glukokortikoiden in den 50er-Jahren konnte die Mortalität um 30\% reduziert werden. Weiter verbessert werden konnte die Prognose durch die Kombination mit verschiedenen steroidsparenden Immunsuppressiva. Bei sehr schweren Verlaufsformen bestehen auch die Möglichkeiten der Plasmapherese, extrakorporalen Photopherese, intravenösen Immunglobulingabe, Immunadsorption und Rituximab-Infusion [3-5].

Zusammenfassend kann man sagen, dass die verschiedenen Behandlungsmöglichkeiten die Mortalität und Morbidität deutlich gebessert haben. Komplikationslose und kurze Therapieverläufe können jedoch nicht garantiert werden.

\section{Literatur}

1 Schmidt E, Zillikens $D$. The diagnosis and treatment of autoimmune blistering skin diseases. Dtsch Arztebl Int 2011; 108: 399-405

2 Lever WF, Schaumburg-Lever G. Immunosuppressants and prednisone in pemphigus vulgaris. Therapeutic results obtained on 63 patients between 1961 and 1975. Arch Dermatol 1977; 113: 1236-1241

3 Hertl M, Zillikens D, Borradori L. Recommendations for the use of rituximab (anti-CD20 antibody) in the treatment of autoimmune bullous skin diseases. J Dtsch Dermatol Ges 2008; 6: 366-373

4 Wollina $U$, Koch A, Hansel G. Rituximab therapy of recalcitrant bullous dermatoses. J Dermatol Case Rep 2008; 2: 4-7

5 Wollina $U$, Lange $D$, Looks A. Short-time extracorporeal photochemotherapy in the treatment of drug-resistant autoimmune bullous diseases. Dermatology 1999; 198: 140-144

\section{Interessenkonflikt}

Die Autoren geben an, dass kein Interessenkonflikt besteht. 